\title{
Deep Video Frame Interpolation Using Cyclic Frame Generation
}

\author{
Yu-Lun Liu, ${ }^{1,2,3}$ Yi-Tung Liao, ${ }^{1,2}$ Yen-Yu Lin, ${ }^{1}$ Yung-Yu Chuang ${ }^{1,2}$ \\ ${ }^{1}$ Academia Sinica, ${ }^{2}$ National Taiwan University, ${ }^{3}$ MediaTek \\ \{yulunliu, queenieliaw\}@cmlab.csie.ntu.edu.tw, yylin@citi.sinica.edu.tw, cyy@csie.ntu.edu.tw
}

\begin{abstract}
Video frame interpolation algorithms predict intermediate frames to produce videos with higher frame rates and smooth view transitions given two consecutive frames as inputs. We propose that: synthesized frames are more reliable if they can be used to reconstruct the input frames with high quality. Based on this idea, we introduce a new loss term, the cycle consistency loss. The cycle consistency loss can better utilize the training data to not only enhance the interpolation results, but also maintain the performance better with less training data. It can be integrated into any frame interpolation network and trained in an end-to-end manner. In addition to the cycle consistency loss, we propose two extensions: motion linearity loss and edge-guided training. The motion linearity loss approximates the motion between two input frames to be linear and regularizes the training. By applying edge-guided training, we further improve results by integrating edge information into training. Both qualitative and quantitative experiments demonstrate that our model outperforms the state-of-the-art methods. The source codes of the proposed method and more experimental results will be available at https://github.com/alex04072000/CyclicGen.
\end{abstract}

\section{Introduction}

High-frame-rate videos with temporally coherent content are preferable, but acquiring such videos often requires higher power consumption and more storage. To compromise between the user experience and the acquiring cost, video frame interpolation, e.g., (Liu et al. 2017; Niklaus, Mai, and Liu 2017b), has drawn increasing attention in the field of computer vision and video processing. It aims at upscaling video frame rates by synthesizing spatially and temporally consistent intermediate frames, and can produce high-quality videos with smooth view transition.

Convolutional neural networks (CNNs) (Krizhevsky, Sutskever, and Hinton 2012) have been employed in many modern methods for video frame interpolation (Niklaus, Mai, and Liu 2017a; 2017b; Liu et al. 2017; Long et al. 2016). These CNN-based methods often integrate motion prediction into video pixel generation, and can work without using hard-to-get dense flow fields as training data. Empowered by CNNs, these methods show excellent abilities

Copyright (C) 2019, Association for the Advancement of Artificial Intelligence (www.aaai.org). All rights reserved.

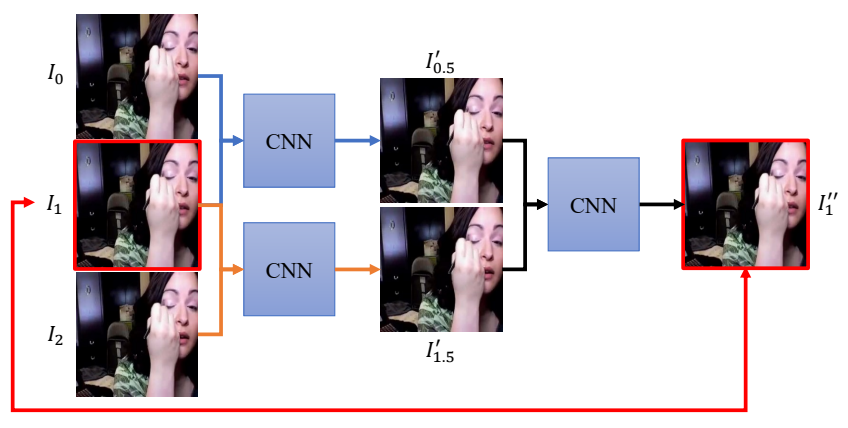

Figure 1: Given three consecutive frames, $I_{0}, I_{1}$, and $I_{2}$, the proposed CNN-based model aims to produce high-quality interpolated frames, $I_{0.5}^{\prime}$ and $I_{1.5}^{\prime}$. To this end, the model maps the given frames to the interpolated frames and then map them back by constructing $I_{1}^{\prime \prime}$ through interpolating between $I_{0.5}^{\prime}$ and $I_{1.5}^{\prime}$. The proposed cycle consistency loss facilitates model learning by enforcing the similarity between $I_{1}$ and $I_{1}^{\prime \prime}$.

to learn the mapping between two consecutive frames and the intermediate frame, and alleviate the problems caused by occlusions and varying lighting conditions. Despite the encouraging progress, video frame interpolation still remains quite challenging: The interpolated frames are usually oversmoothed/blurred and have artifacts (Liang et al. 2017). This situation becomes even worse in image regions with large motions or rich textures.

This paper addresses the aforementioned issues. Our idea is built on the reverse mapping from the interpolated frames to the given frames. Consider a model that is derived to generate the interpolated frame by taking two consecutive frames as inputs. If the interpolated frames are oversmoothed or have artifacts, the reverse mapping is unlikely to be well performed by the same model. Inspired by this observation, we implement forward-backward consistency by introducing the cycle consistency loss for video interpolation. Specifically, the model maps the input frames to the interpolated frames, and then maps them back. The cycle consistency loss enforces the similarity between the input frames and the mapped-back frames. Using the cycle consistency loss, the learned model can implicitly address the 
problems of over-smoothed results and artifacts. In addition to improving performance, this loss constrains the training process, and makes the resultant model more robust even when fewer training data are available. Figure 1 illustrates the basic idea of the cycle consistency loss for video interpolation.

Two extensions, motion linearity loss and edge guided training, are further proposed to address the difficulties of synthesizing frames in the regions with large motions and rich textures, respectively. The motion linearity loss assumes that the view transition is linear with respect to time over consecutive frames in a short period of time. The assumption effectively regularizes network training, and improves the interpolation results. Motivated by the quality degradation of the interpolated frames in highly textured areas, we augment the input with edge information. The generated frames are then improved by better preserving the edge structure. The two extensions complement the cycle consistency loss, and result in considerable performance gains.

We evaluate the performance of our method on three benchmarks, including the UCF101 dataset (Soomro, Zamir, and Shah 2012), a high-quality video, See You Again, (Niklaus, Mai, and Liu 2017b), and the Middlebury optical flow dataset (Baker et al. 2011). The experimental results show that our method performs favorably against the state-of-the-art methods. We also conduct ablation studies to analyze the impact of the proposed cycle consistency loss, motion linearity loss, and edge guided training individually. Additionally, we demonstrate that the cycle consistency loss makes the most of training data, and results in a model robust to the issue of few training data.

\section{Related Work}

This section reviews the topics relevant to this work. We first describe previous methods for video frame interpolation and contrast the proposed method with them. Next, we review the applications of the cycle constraint that the proposed cycle consistency loss roots from.

\section{Video Frame Interpolation}

Conventional methods (Baker et al. 2011; Werlberger et al. 2011; Yu et al. 2013) for frame interpolation usually estimate dense motion correspondences between consecutive frames via stereo matching or optical flow prediction, and can synthesize intermediate frames based on the estimated correspondences. Inheriting from correspondence estimation, these methods induce computation-intensive optimization and are less efficient. Furthermore, these approaches tend to produce artifacts around object boundaries.

CNNs have been shown effective in optical flow estimation such as (Bailer, Taetz, and Stricker 2015; Dosovitskiy et al. 2015; Gadot and Wolf 2016; Güney and Geiger 2016; Teney and Hebert 2016; Tran et al. 2016; Weinzaepfel et al. 2013). These CNN-based methods for flow field prediction need training data in the form of dense correspondences, which are quite hard to annotate. Besides, since their goal is to generate optical flow, the interpolated frames based on optical flow often have artifacts.
Some frame synthesis methods leverage CNNs to directly generate images (Goodfellow et al. 2014) and videos (Vondrick, Pirsiavash, and Torralba 2016; Xue et al. 2016). Thus, they do not use dense correspondences as training data but the ground-truth intermediate frames. However, these methods still suffer from blurred results and artifacts. Liu et al. (Liu et al. 2017) addressed the problem of blurred results by referring to coherent regions of pixels in existing frames and employing a network layer regarding optical flow. Their method makes the synthesized frames sharper, but the problem of artifacts remains unsolved. Other methods (Niklaus, Mai, and Liu 2017a; 2017b) combine motion estimation and frame synthesis into a single convolution step. They estimate spatially-varying kernels for each output pixel, and apply them to input frames for frame interpolation. Despite the effectiveness, these methods need pixelspecific kernel estimation, and consume high computation power and storage usage, especially for high-resolution frame synthesis.

The current state-of-the-art methods (Jiang et al. 2018; Niklaus and Liu 2018) employ CNNs to predict bidirectional optical flow between the input images, and use another CNN model to synthesize interpolated images based on the predicted flow. However, these methods either require additional training data for optical flow estimation or need substantial training time.

Unlike most existing methods that enhance frame interpolation by designing more powerful deep features or architectures, our method mitigates the aforementioned drawbacks by utilizing the cycle consistency loss with two extensions. The advantages of our method are three-fold. First, our method achieves superior performance to the state-ofthe-arts by addressing the issues of blurry results and artifacts. Second, while existing methods require more training data to learn powerful features or networks, we show that our method is more robust against the issue of insufficient training data. Third, using the cycle consistency loss does not increase the model parameters. Thus, both the training and inference costs remain almost unchanged. These nice properties distinguish our method from prior work.

\section{Cycle Constraint}

Using cycle constraints to regularize structured predictions has been explored in the literature. For language translation, it has been shown effective to improve translations by using back translation and reconciliation (Brislin 1970). For visual tracking, enforcing forward-backward consistency is helpful in reaching better results (Sundaram, Brox, and Keutzer 2010). Higher-order cycle consistency has been exploited in diverse vision tasks, such as structure from motion (Zach, Klopschitz, and Pollefeys 2010), 3D shape matching (Huang and Guibas 2013), cosegmentation (Wang, Huang, and Guibas 2013), dense semantic alignment (Zhou et al. 2015; 2016), depth estimation (Godard, Mac Aodha, and Brostow 2017), and imageto-image translation (Zhu et al. 2017). For deep learning, research efforts (Zhou et al. 2016; Godard, Mac Aodha, and Brostow 2017; Zhu et al. 2017) use the concept of cycle consistency to regularize the training of deep models. 


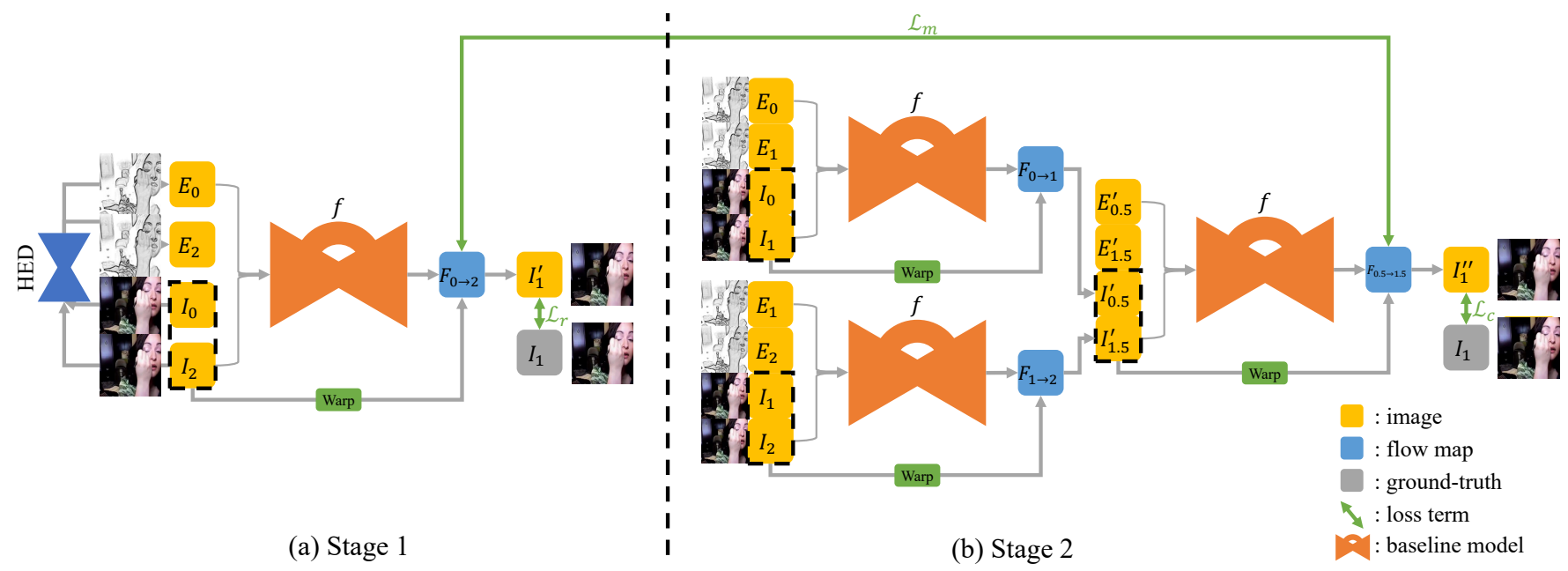

Figure 2: Approach overview. Our approach implements a two-stage optimization process. (a) At the first stage, the baseline model $f$ is pre-trained. (b) At the second stage, the baseline model is duplicated three times. The four models share weights and are fine-tuned by taking into account the proposed cycle consistency loss $\mathcal{L}_{c}$, motion linearity loss $\mathcal{L}_{m}$, and edge guided training. After training, the optimized model $f$ performs video frame interpolation at the inference phase. See text for details.

To the best of our knowledge, this work makes the first attempt to improve video frame interpolation by leveraging cycle consistency. We design a two-stage optimization procedure so that the interpolation model shared by both mapping directions in the cycle constraint can be learned stably. It turns out that our method can greatly improve the quality of interpolation without incurring extra learnable parameters. In addition, the concept of cycle consistency is extended by taking application-specific knowledge into account, and can address the degraded performance in regions with large motions or rich textures.

\section{Proposed Approach}

Given a set of training data $D=\left\{I_{n, 0}, I_{n, 1}, I_{n, 2}\right\}_{n=1}^{N}$, each of which is a triplet containing three consecutive video frames, we aim to learn a deep model that takes two consecutive frames as inputs and can predict their intermediate frame of high quality. Applying the learned model to all consecutive frames of a video doubles its frame rate. Repeatedly applying the model $k$ times upscales the frame rate by a factor of $2^{k}$. For example, given the input frames $S=\left\{I_{0}, I_{1}, I_{2}, \ldots, I_{N}\right\}$, our model first generates frames $S^{\prime}=\left\{I_{0.5}, I_{1.5}, I_{2.5}, \ldots, I_{N-0.5}\right\}$ for $2 \times$ interpolation. Then, it is applied again to $S \cup S^{\prime}$ and produces $S^{\prime \prime}=\left\{I_{0.25}, I_{0.75}, \ldots, I_{N-0.25}\right\}$ for $4 \times$ interpolation.

Our method implements a two-stage training process. Its network architecture is shown in Figure 2. Our method is featured with three newly proposed components, i.e., the cycle consistency loss, motion linearity loss, and edge guided training. The cycle consistency loss boosts the model to produce plausible intermediate frames so that these frames can be used to reversely reconstruct the given frames. The motion linearity loss regularizes the estimation of motions in training. Edge guided training helps preserve the edge structure. We will describe the three components below.

\section{Cycle Consistency Loss $\mathcal{L}_{c}$}

Conventional loss functions, such as the $\ell_{1}$-norm loss, are not fully consistent with human perception when assessing the quality of interpolated frames. Minimizing the $\ell_{1}$ norm loss often leads to over-smoothed frames or artifacts, as shown in Figures 3(a) and 3(b), since these unfavorable effects only yield subtle increase in the loss. Motivated by the observation that the over-smoothed interpolated frames with artifacts cannot well reconstruct the original frames via the same interpolation model, we propose the cycle consistency loss $\mathcal{L}_{c}$, which ensures the quality of reverse frame generation, i.e., generating the original frames with the interpolated frames as input. In this way, these unfavorable effects can be alleviated implicitly by using the cycle consistency loss, as shown in Figure 3(c).

Consider a baseline interpolation model that takes two consecutive frames as inputs and synthesizes the intermediate frame between them. The baseline model can be applied to each triplet $n,\left(I_{n, 0}, I_{n, 1}, I_{n, 2}\right)$, in the training set $D$, where $I_{n, 0}$ and $I_{n, 2}$ serve as the input while $I_{n, 1}$ is the desired output. Since each triplet acts as the input to the model, we sometimes omit the index $n$ for brevity in the paper. Several existing methods, such as AdaConv (Niklaus, Mai, and Liu 2017a) and SepConv (Niklaus, Mai, and Liu 2017b), can serve as the baseline model. In this work, we choose deep voxel flow (DVF) (Liu et al. 2017) as the baseline for its simplicity and good performance. The DVF model learns to synthesize the in-between frame from the input frames by warping the input frames with the predicted flow map and mask. Refer to the original paper of DVF (Liu et al. 2017) for its details.

To stably integrate the cycle consistency loss into model learning and not to increase the number of the learnable parameters in the model, we develop a two-stage training procedure. At the first stage, the baseline model is learned with 
(a)

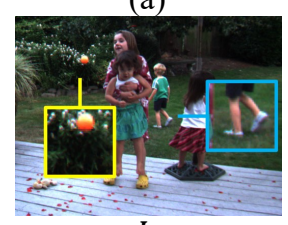

$I_{0}$

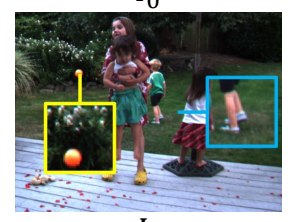

$I_{1}$

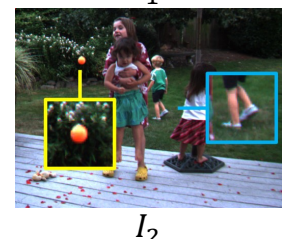

$I_{2}$ (b)
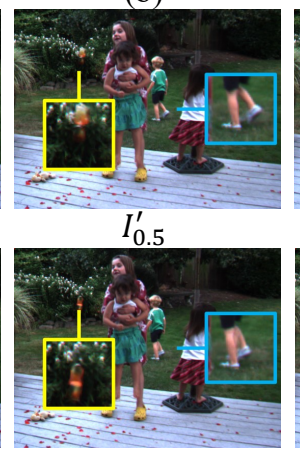

$I_{1.5}^{\prime}$

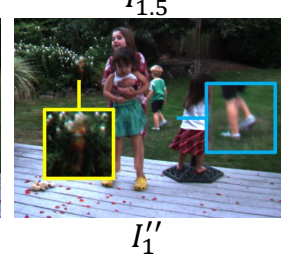

(c)

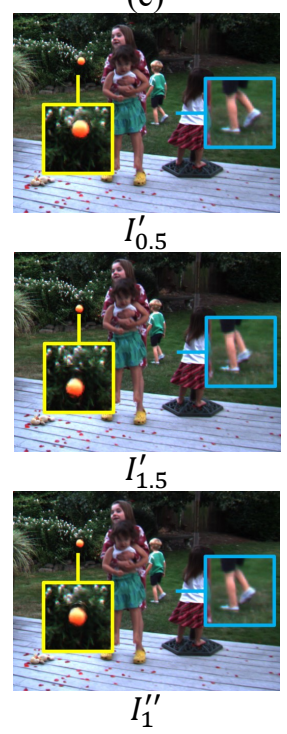

Figure 3: (a) Three input frames, $I_{0}, I_{1}$, and $I_{2}$, for interpolation. (b) Two interpolated frames, $I_{0.5}^{\prime}$ and $I_{1.5}^{\prime}$, by using the $\ell_{1}$-norm loss. The reconstructed $I_{1}$, i.e., $I_{1}^{\prime \prime}$, is obtained by applying the same interpolation model to $I_{0.5}^{\prime}$ and $I_{1.5}^{\prime}$. As can be observed, the issues of artifacts (the region in the yellow box) and over-smoothed results (the region in the blue box) are present in both the interpolated frames and the reconstructed frame. (c) The same figures as those in (b) except the cycle consistence loss is integrated into the loss function for learning the interpolation model. The issues of artifacts and over-smoothed results are greatly alleviated.

each training triplet by using the $\ell_{1}$-norm loss $\mathcal{L}_{r}$ where $r$ stands for reconstruction, i.e.,

$$
\mathcal{L}_{r}=\sum_{n=1}^{N}\left\|f\left(I_{n, 0}, I_{n, 2}\right)-I_{n, 1}\right\|_{1}=\sum_{n=1}^{N}\left\|I_{n, 1}^{\prime}-I_{n, 1}\right\|_{1},
$$

where $f$ is the baseline model.

At the second stage, we duplicate the pre-trained model $f$ three times as shown in Figure 2 for introducing the cycle consistency loss. For a triplet $\left(I_{0}, I_{1}, I_{2}\right)$, the first two duplicated models respectively take $\left(I_{0}, I_{1}\right)$ and $\left(I_{1}, I_{2}\right)$ as input, and generate intermediate frames, $I_{0.5}^{\prime}$ and $I_{1.5}^{\prime}$, which then serve as the input to the third duplicated model to produce the reconstructed $I_{1}$, i.e., $I_{1}^{\prime \prime}$. The cycle consistency loss minimizes the difference between the input frames $I_{1}$ and its reconstructed version $I_{1}^{\prime \prime}$. Specifically, the learnable parameters of the baseline model $f$ and its three duplicated counterparts are shared. At the second stage, the four shared-weight models are trained in an end-to-end manner with the following objective function:

$$
\mathcal{L}=\mathcal{L}_{r}+\mathcal{L}_{c}=\sum_{n=1}^{N}\left\|I_{n, 1}^{\prime}-I_{n, 1}\right\|_{1}+\left\|I_{n, 1}^{\prime \prime}-I_{n, 1}\right\|_{1},
$$

where $I_{n, 1}^{\prime \prime}=f\left(I_{n, 0.5}^{\prime}, I_{n, 1.5}^{\prime}\right), I_{n, 0.5}^{\prime}=f\left(I_{n, 0}, I_{n, 1}\right)$ and

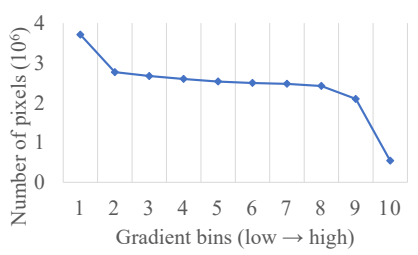

(a)

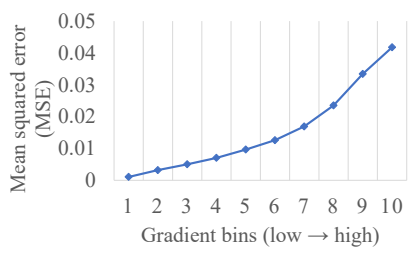

(b)
Figure 4: Interpolation errors of pixels on the DVF testing set. Pixels are divided into ten bins according to their gradient magnitudes. (a) Histogram of gradient magnitudes. (b) Average interpolation error for pixels in each bin. The larger the gradient, the higher the interpolation error.

$I_{n, 1.5}^{\prime}=f\left(I_{n, 1}, I_{n, 2}\right)$ are the generated frames by the three shared-weight models, respectively.

\section{Motion Linearity Loss $\mathcal{L}_{m}$}

Regions with large motions cause dramatic appearance change. Thereby, interpolation on such regions is quite difficult. To address this issue, we assume the time interval between two consecutive frames is short enough so that the motion is linear between the two frames. This assumption helps reduce the uncertainty of motion and alleviate approximation errors in most cases.

Based on the assumption, the motion linearity loss $\mathcal{L}_{m}$ is developed to regularize the optical flow estimation in a selfsupervised fashion. In the two-stage training process, the time intervals between consecutive frames at the first stage are twice as long as those at the second stage. With the motion linearity assumption, the model pre-trained at the first stage generates flow fields with magnitudes twice as large as the duplicated models at the second stage.

In Figure 2, blue blocks represent the estimated flow maps upon which the motion linearity loss $\mathcal{L}_{m}$ is applied. Specifically, this loss is defined by

$$
\mathcal{L}_{m}=\sum_{n=1}^{N}\left\|F_{n, 0 \rightarrow 2}-2 \cdot F_{n, 0.5 \rightarrow 1.5}\right\|_{2}^{2},
$$

where $F$ denotes the flow map and its subscript indicates its data index and the time interval of its input frames.

\section{Edge-guided Training $\mathcal{E}$}

Interpolation on highly textured regions is difficult. To verify this observation, as shown in Figure 4, we applied the DVF model (Liu et al. 2017) and evaluated its performance on the testing set collected in (Liu et al. 2017) by using the metric, mean square error (MSE). By uniformly dividing the interval of gradient magnitudes, we classified pixels into ten bins according to their gradient magnitudes. Figures 4(a) and 4(b) respectively show the histogram of gradient magnitudes and the interpolation error in each bin. It can be observed that pixels with larger gradients tend to have large errors. Inspired by this observation, we implement edge-guided training $\mathcal{E}$ where the edge information is added into the input to the model, and can improve frame interpolation by preserving the edge structure. 
To compute the edge map of an image, we tried several algorithms, including (Canny 1986; Kanopoulos, Vasanthavada, and Baker 1988; Marr and Hildreth 1980; Xie and $\mathrm{Tu}$ 2015). We ended up with using the CNN-based model, holistically-nested edge detection (HED) (Xie and $\mathrm{Tu} 2015$ ), for its performance. In addition, by using CNNbased HED, our method remains end-to-end trainable. As shown in Figure 2, we obtain the edge maps for the two input frames to the model $f$ via HED, and augment them as part of the input to the model.

\section{Implementation Details}

Our method is built on the top of DVF (deep voxel flow) model (Liu et al. 2017) and shares the same setting with it. DVF employs a CNN model to estimate the motion between two consecutive frames and copies pixels from the two frames accordingly to reconstruct the intermediate frame. The optimization is performed with two stages, whose objective functions are respectively specified below

$$
\mathcal{L}_{s_{1}}=\mathcal{L}_{r} \text { and } \mathcal{L}_{s_{2}}=\mathcal{L}_{r}+\lambda_{c} \mathcal{L}_{c}+\lambda_{m} \mathcal{L}_{m}
$$

where $\mathcal{L}_{r}, \mathcal{L}_{c}$, and $\mathcal{L}_{m}$ are defined in Eq. (1), Eq. (2), and Eq. (3), respectively. The weights $\lambda_{c}$ and $\lambda_{m}$ are determined empirically using a validation set. $\lambda_{c}=1$ and $\lambda_{m}=0.1$ are used in all experiments. Every component of our network is differentiable. Thus, our model is end-to-end trainable. The optimization is performed by Adam optimizer (Kingma and $\mathrm{Ba} 2015)$. The batch size is set to 8 , while the learning rate is fixed to 0.0001 during the first stage and reduced to 0.00001 during the second stage optimization.

We had tried to train the model in an end-to-end manner without the first stage. The performance of the network is less reliable due to the lack of the better initialization obtained in the first stage. The result reveals that the initialization from the first stage is beneficial in our case. We had also tried the cycle consistency with a loop of three steps. However, it only resulted in a subtle improvement but made the network much more complex during training. Thus, we only use the two-step cycle in this work.

\section{Experiments}

In this section, we report the datasets used in the experiments, ablation studies and comparisons with the state of the arts. We also discuss the limitations of the proposed method.

\section{Datasets}

We train our model using the training set of the UCF101 dataset (Soomro, Zamir, and Shah 2012). A training video is split into many triplets, each containing three consecutive frames. For each triplet, the middle frame serves as the ground truth while the other two are inputs. To have more challenging samples for training, we select the triplets with more obvious motion by choosing those with lower PSNR values between input frames. Approximately 280,000 triplets are selected to form the training set. For reducing memory consumption, all frames are scaled to the resolution of $256 \times 256$.

\begin{tabular}{lll}
\hline & PSNR & SSIM \\
\hline Baseline (DVF) & 35.89 & 0.945 \\
+ Cycle & $36.71(+0.82)$ & $0.950(+0.005)$ \\
+ Cycle + Motion & $36.85(+0.96)$ & $0.950(+0.005)$ \\
+ Cycle + Edge & $36.86(+0.97)$ & $0.952(+0.007)$ \\
\hline full model & $\mathbf{3 6 . 9 6}(+1.07)$ & $\mathbf{0 . 9 5 3 ( + 0 . 0 0 8 )}$ \\
\hline
\end{tabular}

Table 1: Ablation studies. The numbers in parenthesis indicate improvement against the baseline. (Cycle: cycle consistency loss; Motion: motion linearity loss; Edge: edge-guided training. )

\begin{tabular}{lll}
\hline Training size & Baseline $(\mathrm{DVF})$ & Ours $\left(\mathcal{L}_{c}+\mathcal{L}_{m}\right)$ \\
\hline 1 & 35.98 & 36.85 \\
$1 / 10$ & $35.71(-0.27)$ & $36.83(-0.02)$ \\
$1 / 100$ & $35.43(-0.55)$ & $36.70(-0.15)$ \\
$1 / 1000$ & $34.42(-1.56)$ & $36.10(-0.75)$ \\
\hline
\end{tabular}

Table 2: Evaluation of models with different training data sizes. The average PSNR ( $\mathrm{dB})$ for the UCF101 testing set is reported. The numbers in parenthesis indicate performance drop compared with the same model trained with full data.

We test the proposed network on several datasets, including UCF101 (Soomro, Zamir, and Shah 2012), Middlebury flow benchmark (Baker et al. 2011), and a high-quality YouTube video: See You Again by Wiz Khalifa. For UCF101 and See You Again, in every triple, the first and third frames are used as inputs to predict the second one. The UCF101 testing set consists of 379 sequences provided by (Liu et al. 2017). For the Middlebury flow benchmark, we submit our interpolation results of the eight test sequences to the evaluation website. For quantitative evaluation, we report both Peak Signal-to-Noise Ratio (PSNR) and Structural Similarity Index (SSIM) between the synthesized and real frames.

\section{Ablation Studies}

For understanding the performance of the proposed components, we conduct ablation studies on the UCF101 testing set provided by DVF. As our model is based on DVF, we consider it as the baseline and compare its results with the ones of applying the proposed components, including the cycle consistency loss, motion linearity loss, and edge-guided training, to investigate effectiveness of each component.

Cycle Consistency Loss $\mathcal{L}_{c}$ We first verify whether the idea of cyclic generation improves video interpolation. For that, we compare the results of the baseline with and without the cycle consistency loss $\mathcal{L}_{c}$. The first and second rows in Table 1 clearly indicate that the cycle consistency loss significantly improves the quality of the results, with $0.82 \mathrm{~dB}$ improvement in terms of PSNR. Figure 5 (b) and (c) compare the results without and with $\mathcal{L}_{c}$ on a couple of examples visually. With the help of the cycle consistency loss, the synthesized frames have less visual artifacts (such as the "light streaks" in the baseline results) and exhibit characteristics more similar to the original frames. 


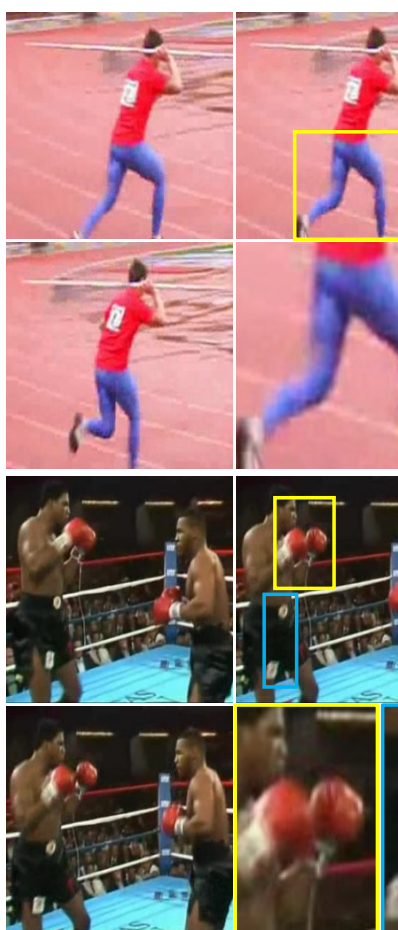

(a)

Inputs

Ground truth

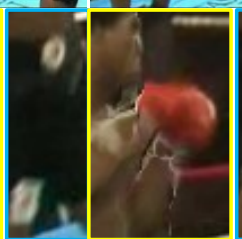

(b)
Baseline (DVF)
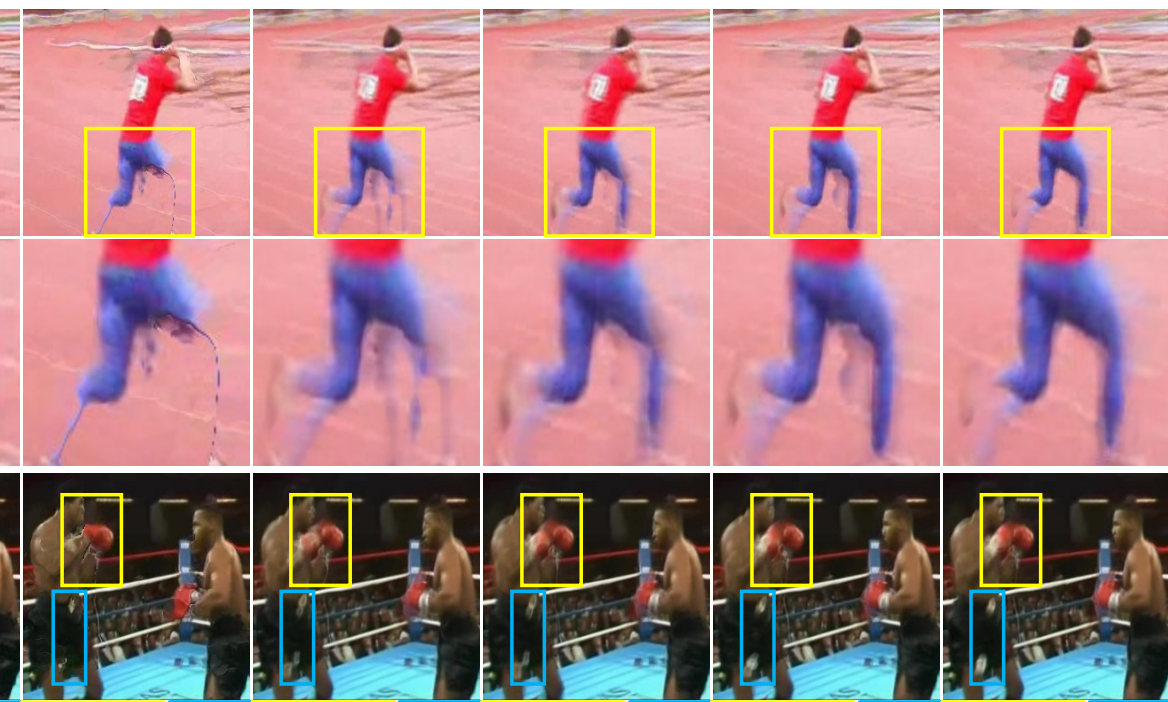

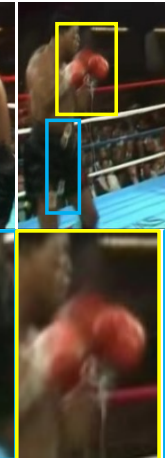

(d)
+ Cycle (c)

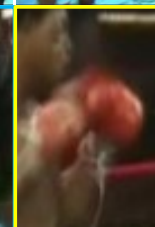

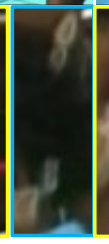

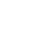

+ Cycle

+ Motion

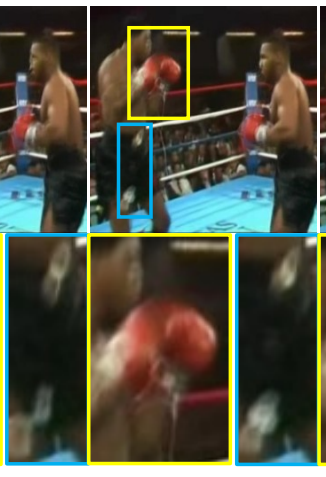

(e)

+ Cycle

+ Edge

Figure 5: Visual comparisons for the ablation studies.

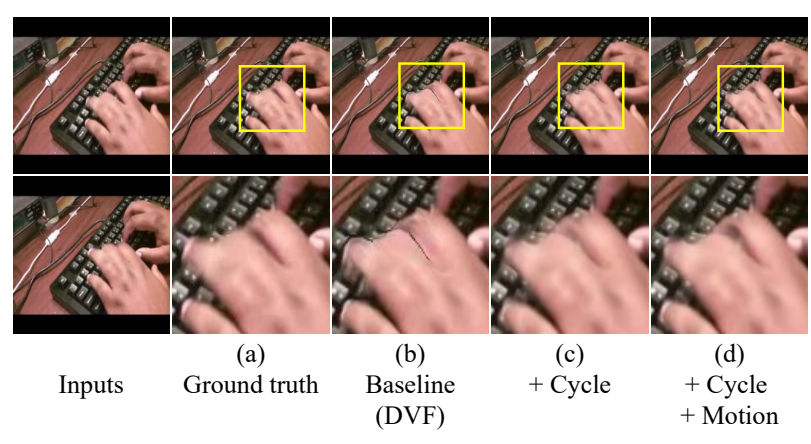

Figure 6: Visual comparisons for the motion linearity loss.

Motion Linearity Loss $\mathcal{L}_{m}$ Table 1 reports that the motion linearity loss brings an additional $0.14 \mathrm{~dB}$ gain on the top of cyclic generation. It is because the assumption of linear motion helps restrict frame synthesis within the subspace with more plausible motion. Figure 5 and Figure 6 give visual examples. As an example, the hand in Figure 6(d) looks sharper with the help of the motion linearity assumption.

Edge-Guided Training $\mathcal{E}$ As shown in Table 1, edgeguided training gains $0.15 \mathrm{~dB}$ to further improve the performance of cyclic generation. It is because the information provided by edge maps helps guide interpolation and improve performance in the areas with strong gradients. Fig-

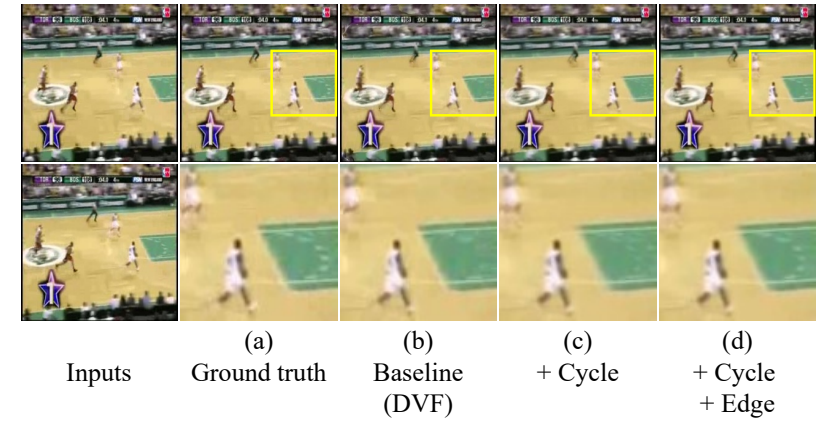

Figure 7: Visual comparisons for the edge-guided training.

ure 5 and Figure 7 show examples where edge-guided training helps. For example, the dashed arc in the free throw lane becomes visible in Figure 7(d) with the help of edge-guided training.

Impact of the Number of Training Samples Our cyclic frame generation method uses the generated frames as training data for the second-stage training. It has the potential to utilize the data more effectively. We conduct experiments to verify the point by varying the amount of training data. The full UCF101 training data contain approximately 280,000 triplets. We reduce the training data to $1 / 10,1 / 100$ and $1 / 1000$ of the full data by randomly sampling triplets. Table 2 reports the performance of the baseline method (DVF) 


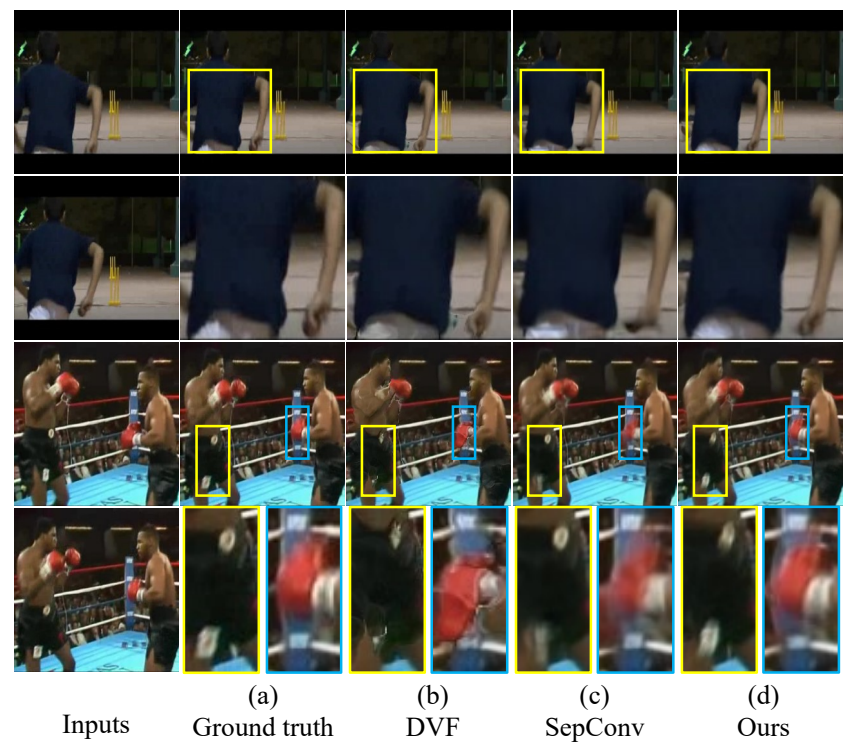

Figure 8: Visual comparisons for examples from UCF101.

\begin{tabular}{lccccc}
\hline & \multicolumn{2}{c}{ UCF101 } & & \multicolumn{2}{c}{ See You Again } \\
\cline { 2 - 3 } \cline { 6 - 7 } & PSNR & SSIM & & PSNR & SSIM \\
\hline DVF & 35.89 & 0.945 & & 40.15 & 0.958 \\
SepConv & 36.49 & 0.950 & & 41.01 & $\mathbf{0 . 9 6 8}$ \\
\hline Ours & $\mathbf{3 6 . 9 6}$ & $\mathbf{0 . 9 5 3}$ & & $\mathbf{4 1 . 6 7}$ & $\mathbf{0 . 9 6 8}$ \\
\hline
\end{tabular}

Table 3: Quantitative comparisons on the UCF101 testing set and the high-quality video See You Again.

and our method $\left(\mathcal{L}_{c}+\mathcal{L}_{m}\right)$ with different amounts of training data. It is clear that the proposed method maintains performance better with less training data. For example, when using $1 / 10$ of the training data, our method almost performs as well as the one using full data by only dropping $0.02 \mathrm{~dB}$. For the same amount of data, the baseline method has already dropped $0.27 \mathrm{~dB}$. When using only $1 / 1000$ of the training data (meaning merely 280 triplets for training), our method can still keep very good performance $(36.10 \mathrm{~dB})$, even better than the baseline method trained with full data (35.98dB). The experiment shows that our method utilizes the training data very well. It suffers less from over-fitting and is robust even with little training data.

\section{Comparisons with State-of-the-Art Methods}

We compare our method mainly with two state-ofthe-art methods, separable adaptive convolution (SepConv) (Niklaus, Mai, and Liu 2017b) and deep voxel flow (DVF) (Liu et al. 2017), with publicly available executables. While evaluating on the Middlebury flow benchmark, we also compare with the top performance methods listed in the benchmark website. The supplementary video shows interpolation results of our method for a $8 \times$ frame rate.

UCF101 For UCF101, we compute both PSNR and SSIM using the motion masks provided by (Liu et al. 2017). As reported in Table 3, for UCF101, our method outperforms both
DVF and SepConv, by $1.07 \mathrm{~dB}$ and $0.47 \mathrm{~dB}$ in PSNR respectively. Figure 8 shows visual comparisons of these methods.

See You Again For testing generalization of the model, we apply the model trained on UCF101 to a high-quality YouTube video, See You Again. It has 5,683 frames with the resolution $960 \times 540$. We take odd-number frames as inputs and synthesize the even-number frames. The right part of Table 3 reports PSNR and SSIM values of compared methods on See You Again. Our method outperforms the others.

Middlebury To better accommodate the large appearance difference between UCF101 and Middlebury, we finetune our model using the training set provided by Middlebury, which contains only 168 frames. Table 4 reports the interpolation errors (IE) of our model and five topperformance methods in Middlebury's list on the eight testing sequences of the benchmark. The compared methods include (1) synthesis-based methods which directly generate interpolated frames: CtxSyn (Niklaus and Liu 2018), SepConv (Niklaus, Mai, and Liu 2017b), and SuperSlomo (Jiang et al. 2018); and flow-based methods which utilize optical flows for frame interpolation: MDP-Flow2 (Xu, Jia, and Matsushita 2012) and DeepFlow (Weinzaepfel et al. 2013). At the time when the paper is submitted, CtxSyn ranks the first and all five compared methods are among top six. Our model achieves the best average performance and owns the best records for 5 out of all 8 sequences. Note that the last four sequences contain real images. It indicates that our method performs particularly well on real-scene sequences. Fine-tuning with 168 frames also shows that our approach performs well with little training data. Figure 9 shows visual comparisons on examples from the Middlebury benchmark.

\section{Limitations}

Although our method provides good improvement, it has some limitations. First, dealing with the areas with large motions remains challenging to our method. As shown in Figures 5 and 9, although the cycle consistency and motion linearity losses jointly alleviate the interpolation errors, there are still interpolation errors in the regions with large motions. Second, the motion linearity loss assumes linear motion, but the linearity assumption may not hold in videos at very low frame rates, which likely would cause interpolation errors. In addition, our method performs repeated interpolation. Hence, it only upscales the frame rate by a factor of $2^{N}$, instead of an arbitrary frame rate that a user desires.

\section{Conclusion}

We present a novel loss, the cycle consistency loss, which can be integrated with existing video frame interpolation methods and trained in an end-to-end manner. By ensuring the interpolated frames can be used to reconstruct the input frames, our method synthesizes more plausible frames possessing similar characteristics with the original frames. Additionally, we propose two extensions, motion linearity loss and edge guided training, to regularize the training procedure and further improve model performance. The proposed 


\begin{tabular}{|c|c|c|c|c|c|c|c|c|c|c|c|c|c|c|c|c|c|c|c|c|c|c|c|c|c|c|c|}
\hline & \multicolumn{3}{|c|}{ AVERAGE } & \multicolumn{3}{|c|}{ Mequon } & \multicolumn{3}{|c|}{ Schefflera } & \multicolumn{3}{|c|}{ Urban } & \multicolumn{3}{|c|}{ Teddy } & \multicolumn{3}{|c|}{ Backyard } & \multicolumn{3}{|c|}{ Basketball } & \multicolumn{3}{|c|}{ Dumptruck } & \multicolumn{3}{|c|}{ Evergreen } \\
\hline & all & disc. & unt. & all & disc. & unt. & all & disc. & unt. & all & disc. & unt. & all & disc. & unt. & all & disc. & unt. & all & disc. & unt. & all & disc. & unt. & all & disc. & unt. \\
\hline Ours & 4.20 & 6.16 & 1.97 & 2.26 & 3.32 & 1.42 & 3.19 & 4.01 & 2.21 & 2.76 & 4.05 & 1.62 & 4.97 & 5.92 & 3.79 & 8.00 & 9.84 & 3.13 & 3.36 & 5.65 & 2.17 & 4.55 & 9.68 & 1.42 & 4.48 & 6.84 & 1.52 \\
\hline CtxSyn & 5.28 & 8.00 & 2.19 & 2.24 & 3.72 & 1.04 & 2.96 & 4.16 & 1.35 & 4.32 & 3.42 & 3.18 & 4.21 & 5.46 & 3.00 & 9.59 & 11.9 & 3.46 & 5.22 & 9.76 & 2.22 & 7.02 & 15.4 & 1.58 & 6.66 & 10.2 & 1.69 \\
\hline SuperSlomo & 5.31 & 8.39 & 2.12 & 2.51 & 4.32 & 1.25 & 3.66 & 5.06 & 1.93 & 2.91 & 4.00 & 1.41 & 5.05 & 6.27 & 3.66 & 9.56 & 11.9 & 3.30 & 5.37 & 10.2 & 2.24 & 6.69 & 15.0 & 1.53 & 6.73 & 10.4 & 1.66 \\
\hline SepConv & 5.61 & 8.74 & 2.33 & 2.52 & 4.83 & 1.11 & 3.56 & 5.04 & 1.90 & 4.17 & 4.15 & 2.86 & 5.41 & 6.81 & 3.88 & 10.2 & 12.8 & 3.37 & 5.47 & 10.4 & 2.21 & 6.88 & 15.6 & 1.72 & 6.63 & 10.3 & 1.62 \\
\hline MDP-Flow2 & 5.83 & 9.69 & 2.15 & 2.89 & 5.38 & 1.19 & 3.47 & 5.07 & 1.26 & 3.66 & 6.10 & 2.48 & 5.20 & 7.48 & 3.14 & 10.2 & 12.8 & 3.61 & 6.13 & 11.8 & 2.31 & 7.36 & 16.8 & 1.49 & 7.75 & 12.1 & 1.69 \\
\hline DeepFlow & 5.97 & 9.79 & 2.05 & 2.98 & 5.67 & 1.22 & 3.88 & 5.78 & 1.52 & 3.62 & 5.93 & 1.34 & 5.39 & 7.20 & 3.17 & 11.0 & 13.9 & 3.63 & 5.91 & 11.3 & 2.29 & 7.14 & 16.3 & 1.49 & 7.80 & 12.2 & 1.70 \\
\hline
\end{tabular}

Table 4: Quantitative comparisons on the Middlebury benchmark. The table reports the interpolation errors. disc.: regions with discontinuous motion. unt.: textureless regions.

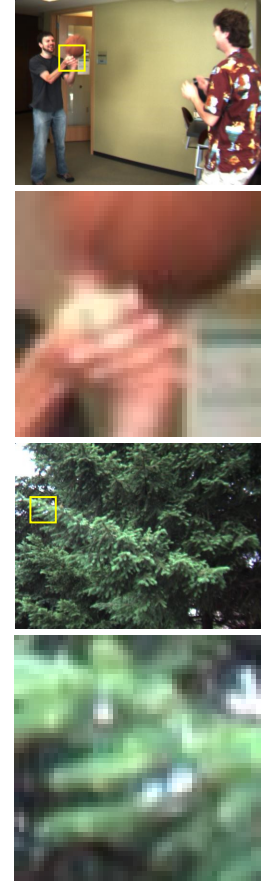

(a) Ground truth

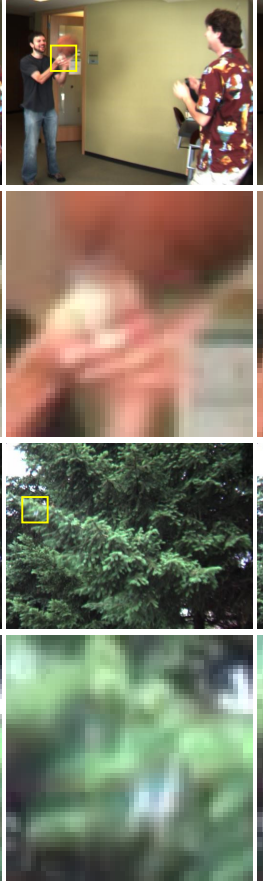

(b) CtxSyn

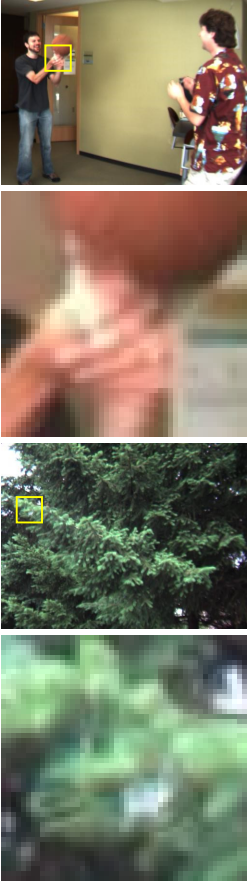

(c) SuperSlomo

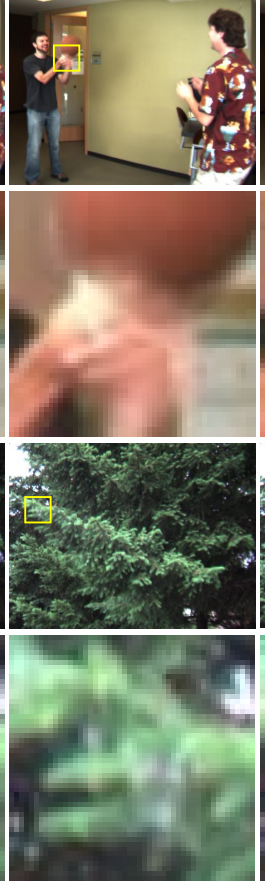

(d) SepConv

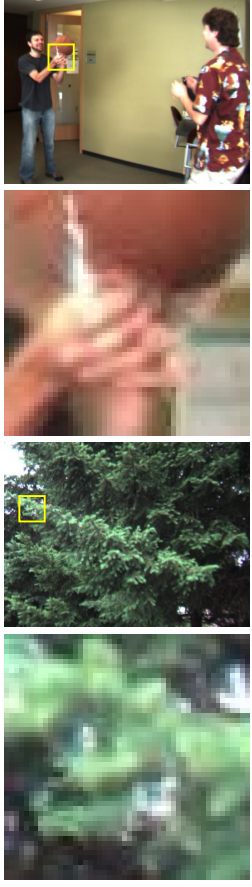

(e) MDP-Flow2

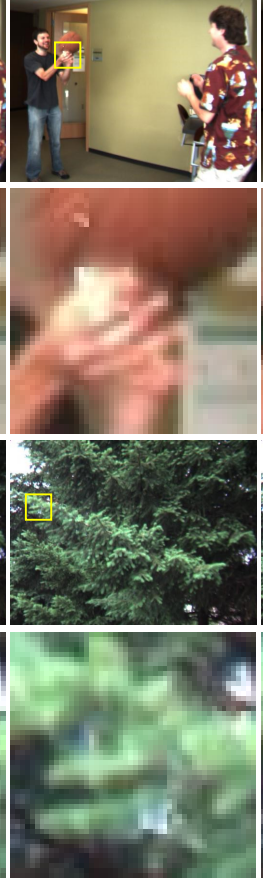

(f) DeepFlow2

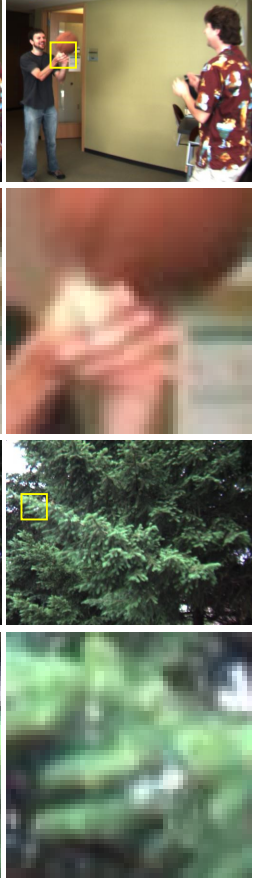

(g) Ours

Figure 9: Visual comparisons of our method and five competing methods on examples from the Middlebury benchmark.

approach better utilizes the training data, not only enhancing the interpolation results, but also reaching better performance with less training data.

\section{Acknowledgments}

This work was supported in part by Ministry of Science and Technology (MOST) under grants MOST 107-2628-E-001005-MY3, MOST107-2221-E-002-147-MY3, and MOST Joint Research Center for AI Technology and All Vista Healthcare under grant 107-2634-F-002-007.

\section{References}

Bailer, C.; Taetz, B.; and Stricker, D. 2015. Flow fields: Dense correspondence fields for highly accurate large displacement optical flow estimation. In Proceedings of IEEE ICCV.

Baker, S.; Scharstein, D.; Lewis, J.; Roth, S.; Black, M. J.; and Szeliski, R. 2011. A database and evaluation methodology for optical flow. International Journal of Computer Vision 92(1):1-31.
Brislin, R. W. 1970. Back-translation for cross-cultural research. Journal of cross-cultural psychology 1(3):185-216. Canny, J. 1986. A computational approach to edge detection. IEEE Transactions on Pattern Analysis and Machine Intelligence 8(6):679-698.

Dosovitskiy, A.; Fischer, P.; Ilg, E.; Hausser, P.; Hazirbas, C.; Golkov, V.; van der Smagt, P.; Cremers, D.; and Brox, T. 2015. FlowNet: Learning optical flow with convolutional networks. In Proceedings of IEEE ICCV.

Gadot, D., and Wolf, L. 2016. PatchBatch: a batch augmented loss for optical flow. In Proceedings of IEEE CVPR.

Godard, C.; Mac Aodha, O.; and Brostow, G. J. 2017. Unsupervised monocular depth estimation with left-right consistency. In Proceedings of IEEE CVPR.

Goodfellow, I.; Pouget-Abadie, J.; Mirza, M.; Xu, B.; Warde-Farley, D.; Ozair, S.; Courville, A.; and Bengio, Y. 2014. Generative adversarial nets. In Proceedings of NIPS. Güney, F., and Geiger, A. 2016. Deep discrete flow. In Proceedings of ACCV.

Huang, Q.-X., and Guibas, L. 2013. Consistent shape maps via semidefinite programming. In Proceedings of the 
Eleventh Eurographics/ACMSIGGRAPH Symposium on Geometry Processing.

Jiang, H.; Sun, D.; Jampani, V.; Yang, M.-H.; LearnedMiller, E.; and Kautz, J. 2018. Super slomo: High quality estimation of multiple intermediate frames for video interpolation. In Proceedings of IEEE CVPR.

Kanopoulos, N.; Vasanthavada, N.; and Baker, R. L. 1988. Design of an image edge detection filter using the Sobel operator. IEEE Journal of Solid-State Circuits 23(2):358-367.

Kingma, D. P., and Ba, J. 2015. Adam: A method for stochastic optimization. In Proceedings of ICLR.

Krizhevsky, A.; Sutskever, I.; and Hinton, G. E. 2012. ImageNet classification with deep convolutional neural networks. In Proceedings of NIPS.

Liang, X.; Lee, L.; Dai, W.; and Xing, E. P. 2017. Dual motion GAN for future-flow embedded video prediction. In Proceedings of IEEE ICCV.

Liu, Z.; Yeh, R.; Tang, X.; Liu, Y.; and Agarwala, A. 2017. Video frame synthesis using deep voxel flow. In Proceedings of IEEE ICCV.

Long, G.; Kneip, L.; Alvarez, J. M.; Li, H.; Zhang, X.; and Yu, Q. 2016. Learning image matching by simply watching video. In Proceedings of ECCV.

Marr, D., and Hildreth, E. 1980. Theory of edge detection. Proc. R. Soc. Lond. B 207:187-217.

Niklaus, S., and Liu, F. 2018. Context-aware synthesis for video frame interpolation. In Proceedings of IEEE CVPR.

Niklaus, S.; Mai, L.; and Liu, F. 2017a. Video frame interpolation via adaptive convolution. In Proceedings of IEEE CVPR.

Niklaus, S.; Mai, L.; and Liu, F. 2017b. Video frame interpolation via adaptive separable convolution. In Proceedings of IEEE ICCV.

Soomro, K.; Zamir, A. R.; and Shah, M. 2012. UCF101: A dataset of 101 human actions classes from videos in the wild. Technical Report CRCV-TR-12-01, University of Central Florida.

Sundaram, N.; Brox, T.; and Keutzer, K. 2010. Dense point trajectories by GPU-accelerated large displacement optical flow. In Proceedings of ECCV.

Teney, D., and Hebert, M. 2016. Learning to extract motion from videos in convolutional neural networks. In Proceedings of $A C C V$.

Tran, D.; Bourdev, L.; Fergus, R.; Torresani, L.; and Paluri, M. 2016. Deep end2end voxel2voxel prediction. In Proceedings of IEEE CVPR Workshops.

Vondrick, C.; Pirsiavash, H.; and Torralba, A. 2016. Generating videos with scene dynamics. In Proceedings of NIPS.

Wang, F.; Huang, Q.; and Guibas, L. J. 2013. Image cosegmentation via consistent functional maps. In Proceedings of IEEE ICCV.

Weinzaepfel, P.; Revaud, J.; Harchaoui, Z.; and Schmid, C. 2013. DeepFlow: Large displacement optical flow with deep matching. In Proceedings of IEEE ICCV.
Werlberger, M.; Pock, T.; Unger, M.; and Bischof, H. 2011. Optical flow guided TV-L1 video interpolation and restoration. In Proceedings of IEEE CVPR Workshops.

Xie, S., and Tu, Z. 2015. Holistically-nested edge detection. In Proceedings of IEEE ICCV.

Xu, L.; Jia, J.; and Matsushita, Y. 2012. Motion detail preserving optical flow estimation. IEEE Transactions on Pattern Analysis and Machine Intelligence 34(9):1744-1757.

Xue, T.; Wu, J.; Bouman, K.; and Freeman, B. 2016. Visual dynamics: Probabilistic future frame synthesis via cross convolutional networks. In Proceedings of NIPS.

Yu, Z.; Li, H.; Wang, Z.; Hu, Z.; and Chen, C. W. 2013. Multi-level video frame interpolation: Exploiting the interaction among different levels. IEEE Transactions on Circuits and Systems for Video Technology 23(7):1235-1248.

Zach, C.; Klopschitz, M.; and Pollefeys, M. 2010. Disambiguating visual relations using loop constraints. In Proceedings of IEEE CVPR.

Zhou, T.; Jae Lee, Y.; Yu, S. X.; and Efros, A. A. 2015. FlowWeb: Joint image set alignment by weaving consistent, pixel-wise correspondences. In Proceedings of IEEE CVPR.

Zhou, T.; Krahenbuhl, P.; Aubry, M.; Huang, Q.; and Efros, A. A. 2016. Learning dense correspondence via 3D-guided cycle consistency. In Proceedings of IEEE CVPR.

Zhu, J.-Y.; Park, T.; Isola, P.; and Efros, A. A. 2017. Unpaired image-to-image translation using cycle-consistent adversarial networks. In Proceedings of IEEE ICCV. 